This document is the author's final manuscript of

F. Tateo, M. Collet, M. Ouisse and K.A. Cunefare: Design variables for optimizing adaptive metacomposite made of shunted piezoelectric patches distribution. Journal of Vibration and Control, 2014.

This paper has been published by SAGE Publications and can be found at http://dx.doi.org/10.1177/1077546314545100 


\title{
Design Variables for Optimizing Adaptive Metacomposite Made of Shunted Piezoelectric Patches Distribution
}

\author{
F. Tateo, M.Collet ${ }^{\dagger}$ M. Ouisse* ${ }^{*}$ K.A. Cunefare ${ }^{\ddagger}$
}

September 4, 2014

\begin{abstract}
A two-dimensional array of piezoelectric transducer (PZT) shunted on negative capacitance circuit is designed and applied to achieve broadband vibration reduction of a flexible plate over tunable frequency bands. Each surface-bonded patch is connected to a single independent negative capacitance synthetic circuit.

A finite element-based design methodology is used to predict and optimize the attenuation properties of the smart structure. The predictions are then experimentally validated by measuring the harmonic response of the plate and evaluating some derived quantity such as the loss factor and the kinetic energy ratio. The validated model is finally used to explore different configurations with the aim of defining some useful design criteria.

The results obtained clearly show how the proposed strategy represents a robust and effective solution for the control of vibrations in complex structures.
\end{abstract}

\section{Keyword}

Metacomposites, Semi-active control, Wave propagation, Negative capacitance circuit, Piezoelectricity, Energy diffusion.

\section{Introduction}

Shunted piezoelectric patches is a promising control technique for vibration suppression of flexible structures. The technique was first introduced by Forward (1979) who used piezoelectric elements with active feedback circuits to significantly reduce the mechanical response of a membrane mirror. Contrary to active control, the only external element to be used is a passive electrical network that is directly connected to the electrodes of the piezoelectric device.

With this approach, the sensing element is not needed and the use of a passive network guarantees the stability of the coupled system. From the theoretical point of view the first analytical formulation of such passive shunt networks was provided by Hagood and von Flotow (1991). According

\footnotetext{
*Femto-st UMR-CNRS 6174, 24 chemin de l'Epitaphe, 25000 Besancon, France.

${ }^{\dagger}$ LTDS UMR-CNRS 5513, Ecole Centrale de Lyon, 36 avenue Guy de Collongue, 69134 Ecully, France.

${ }^{\ddagger}$ Woodruff School of Mechanical Engineering, Georgia Institute of Technology, Atlanta, GA, USA.
} 
to their interpretation the piezoelectric patch shunted through a resistive inductive circuit acts as a tuned mass damper adjusted at the resonance frequency of the circuit.

Since then, more complex shunting circuits have been investigated to extend the control system's effectiveness over broader frequency bands. For example, a multi-mode technique has been proposed by $\mathrm{Wu}$ (1999) who employed a series of blocking, inductive-capacitive circuits in parallel with an RL shunt circuit designed to attenuate a specific resonance frequency. Hollkamp (1994) developed a different multi-mode circuit based on RL series components in parallel with $\mathrm{n}$ similar branches, one for each mode to be suppressed. Each branch consists of a RLC network so that if only one mode is damped this circuit reduces to the one proposed by Hagood and Von Flotow.

However, no closed form tuning solution has been found for this technique and numerical optimization is the only way to simultaneously determine the values for the electrical components. For the control of a large number of modes, numerical optimization may result in a complicated procedure and the implementation of the circuit may become a difficult task due to the high order of the system. Fleming et al. (2000) proposed a different approach to multimodal control employs complex control networks featuring variable synthetic impedances, however, the resulting active control configuration tend to be difficult to implement.

More recently, the idea of distributed vibration absorbers was introduced by Alessandroni et al. (2002) who proposed to use distributed piezoelectric transducers to couple mechanical structures with distributed electric networks. The key concept is to use interconnected distributed circuits coupled with elastic structures to obtain wide-band electromechanical energy exchange. In Alessandroni et al. (2002) electric analogs of transversely vibrating beams and plates are presented and coupled with distributed piezoelectric elements by means of homogenized models. The performance of different circuits interconnections is studied by Maurini et al. (2004). The numerical results are associated with the need for a minimum number of electromechanical modules per unit length to control the oscillations for given wave-numbers. This may result in electrical networks of significant complexity.

Another important contribution to this subject is offered by Andreaus et al. (2004) where a PiezoElectroMechanical (PEM) beam with distributed piezoelectric transducers interconnected to an analog circuit is analyzed. In this paper a rational analysis of this smart device is provided along with a precious information about the limits and the advantages of such passive control strategy. A practical implementation can be found in dell'Isola et al. (2004) who provided experimental results of such a damping concept on a beam featuring resistive and resistive inductive interconnected circuits.

In literature, over the years, other different solutions have been proposed. For example it worth to mention the method based on an array of independently-shunted piezoelectric patches distributed over the whole structure. This results in a periodic configuration, where equivalent sources of mechanical impedance mismatch are introduced through proper tuning of the shunting circuits. The selection of this configuration is driven by the unique properties of broadband wave attenuation of periodic structures.

A periodic structure is defined as heterogeneous domain characterized by a recursive pattern obtained through the translation in space of a fundamental unit cell. The dynamics of periodic engineering structures has been mainly investigated in the past by Mead (1996) and his co-workers, who highlighted how in this class of assemblies wave propagation occurs only over specific frequency 
bands known as pass bands, while significant attenuation occurs over other regions of the frequency domain called stop bands or band gaps. The capabilities of controlling the impedance of a shunted piezoelectric patch are used to modify the ability of the periodic structure to transmit waves.

The propagation of waves in smart periodic rods and axisymmetric shells was studied by Thorp et al. (2005) who used RL piezoelectric shunts to passively control the vibration field. Recently Spadoni et al. (2009) extended the concept to flat plates demonstrating that the width and location of stop bands in the frequency domain can be shaped through the tunable characteristics of periodic RL shunts.

These techniques exploit the energy exchange mechanism established between the controlled structure and a resonating element to control its dynamic behavior. The effectiveness of this control strategy depends on the accurate knowledge of the modes of the controlled structures, for small variation of the tuning parameter of the external circuit a degradation in performance is usually observed. Moreover the RL control strategy suffer from another limitation, namely the impossibility to control the structure over a wide frequency band.

Vidoli and dell'Isola (2001) proposed to control the vibrations in plates by means of a set of uniformly distributed electrically interconnected actuators. In their work they have shown how the correct selection of the circuital parameters could increase the electromechanical coupling between the structure and the two-dimensional electrical network. An internal resonance phenomenon, between structural modes and electric modes, is induced to obtain the maximum control efficiency. A possible alternative is the use of negative capacitance circuits. Park and Palumbo (2004) developed a shunting strategy based on a fixed negative capacitance circuit with the objective of simplifying the electrical network. The performances of a negative capacitance circuit were also investigated by Park and Baz (2005), who showed that the negative capacitance shunt is an effective mean to control structural vibrations over broader frequency bands. Normally this function is obtained by using electronic circuits able to synthetize the negative capacitance effect. From this point of view this shunt strategy can be assimilated to an active feedback control that produces instabilities if improperly tuned as shown by Behrens et al. (2003).

In this paper, an array of piezoelectric patches shunted to a set of negative capacitance circuits and periodically arranged over a limited region of the surface of a two-dimensional waveguide is considered. This array of patches is intended to serve as an active interface between regions of the waveguide, formed from a plate. This active interface allows modification of the scattering properties of the waveguide in terms of reflected and absorbed energy. This controlling capability is obtained by correctly tuning the parameters of the external circuit by which almost arbitrary effective structural impedance may be obtained. The selection of the electric component is made according to the methodologies developed by Collet et al. (2012a) and subsequently extended to more complicated cases involving fluid-structure interaction in Collet et al. (2012b). Experimental validation of this approach has been made on a beam by Beck et al. (2011) and subsequently extended to a flexible plate completely covered by piezoelectric patches by Casadei et al. (2010). A finite element-based design methodology is used to predict the attenuation properties of the smart structure. The predictions are then experimentally validated by measuring the harmonic response of the plate and evaluating some derived quantity such as the loss factor and the kinetic energy ratio. The loss factor and the kinetic energy ratio (calculated in each subdomain of the plate) are used to qualify the absorbing and reflecting properties of the metacomposite. The validated model is also used to explore different configurations with the aim of defining some general design criteria and then assess the robustness of the overall system. 


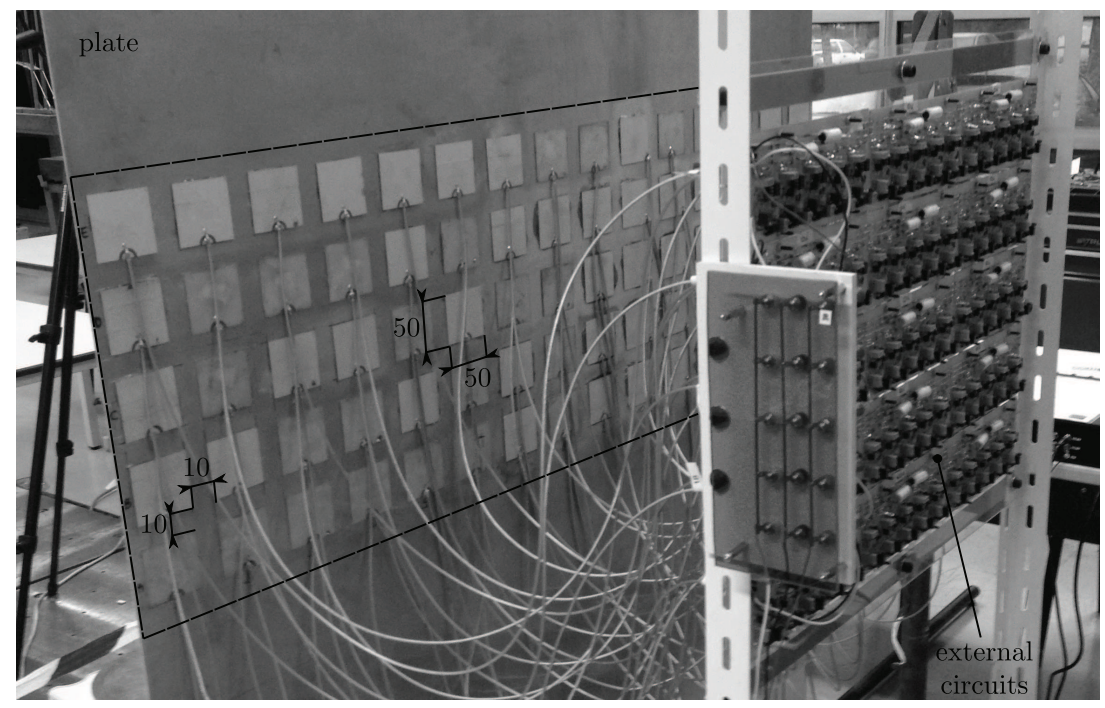

Figure 1: plate with 75 element piezoelectric patch array comprising an active interface, connected to bank of 75 individual negative impedance shunt control circuits. Patch dimensions and spacing annotated on photograph.

The remainder of this paper is organized into four sections. The first section provides a review of the design of the plate, piezoelectric array, and shunt circuits for the control system applied to the patches in the piezoelectric array. The second section describes the numerical model used to investigate the system. The third section presents the experimental and numerical results. Finally, the last section summarizes the major findings presented in this paper and provides recommendations for future investigation.

\section{Metacomposite Design}

\subsection{System Description}

The basic structure of the metacomposite comprises an aluminum plate and array of piezoelectric patches as depicted in Figure 1. The plate is suspended from a rigid frame by metallic wires in order to approximate free-free boundary conditions. The plate is equipped with 75 piezoelectric patches (Ferroperm Industries PZ 26 series) arranged in a regular $15 \times 5$ array. The system's configuration depicted in Figure 1 is associated to the label A, along the paper other configuration are also studied by modifying some geometrical parameter as summarized in Table 1 . The parameter $L_{p z}$

\begin{tabular}{lllllllll}
\hline type & patch \# & $L_{p z}$ & $l_{p z}$ & $h_{p z}$ & $p_{x}$ & $p_{y}$ & $L_{\text {int }}$ & $l_{\text {int }}$ \\
\hline A & $15 \times 5$ & 50 & 50 & 0.5 & 10 & 10 & 1050 & 850 \\
B & $15 \times 5$ & 50 & 150 & 0.5 & 10 & 10 & 1050 & 350 \\
C & $20 \times 7$ & 50 & 30 & 0.5 & 10 & 10 & 1050 & 350 \\
\hline
\end{tabular}

Table 1: Smart interface's configurations and piezoelectric ceramic's dimensions expressed in $m m$. 

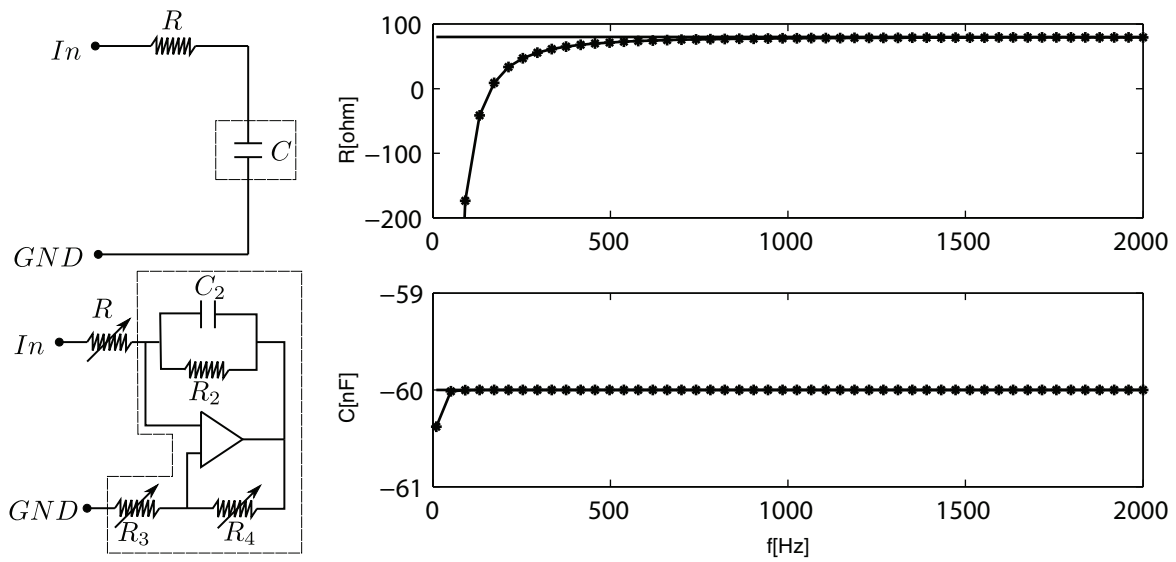

Figure 2: Ideal vs. real-life circuit. On the left the basic architecture for both circuits is presented. On the right the circuit's impedance is depicted in terms of equivalent resistance (top) and equivalent capacitance (bottom): the solid line represent the ideal circuit; the solid line with markers the real-life circuit.

$l_{p z}$ and $h_{p z}$ represent respectively the length of the piezoelectric ceramic along the $x, y$ and $z$ axes; $p_{x}$ and $p_{y}$ symbolize the distance between two adjacent piezoelectric ceramic in $x$ and $y$ direction; $L_{\text {int }}$ and $l_{\text {int }}$ are associated to the extension of the active interface.

The length and the depth of the patch array were selected based on consideration of their size relative to the shortest structural wavelength of interest. The array was intended to be effective up to a frequency of $5000 \mathrm{~Hz}$. The plate's dispersion relation for the $A_{0}$ flexural mode at $5000 \mathrm{~Hz}$ has a wavelength of approximately $30 \mathrm{~cm}$. With patches having dimensions of $50 \times 50 \mathrm{~mm}$, there will be no charge cancellation on an individual patch as would occur if the patch dimensions were comparable to a wavelength. Further, the depth of the patch array is $350 \mathrm{~mm}$ such that it spans more than a wavelength at $5000 \mathrm{~Hz}$. Since the array elements are uncoupled electronically, each element within the array would still function at the highest frequency of interest, even though the entire array depth would be comparable to a wavelength.

The thickness of the patch was selected considering the constraints of the electric circuit and the nature of the control technique. Different authors have shown that the best controlling effect is obtained when the negative impedance shut circuit is tuned close to the inherent patch capacitance (Livet et al. (2011)). This property strongly depends on the material properties and the geometry of the patch. Once the material properties and the two dimensions of the piezoelectric patch are chosen, the only parameter which remains for selection is the thickness. Thin patches exhibit larger values of intrinsic capacitance which would yield increased control, however, this parameter cannot be reduced indefinitely due to the weakening of the piezoelectric patch itself. For these reasons, a thickness of $0.5 \mathrm{~mm}$ was selected.

The results presented in Collet et al. (2012a) provide an approach for optimization of the external shunt circuit in terms of the real and the imaginary parts of the synthetic impedance. The authors identified two possible behaviors depending on the criterion used in the optimization. The first one is the minimization of the velocity of the energy propagation in the region of the active array which limits the propagation of the waves beyond the active interface. This optimal circuit is able to modify the transmission properties of the plate by increasing the energy reflected from the active 
interface. The optimal controlling impedance for this approach requires a negative capacitance with a relatively small negative resistance. As shown in Collet et al. (2012a) the theoretical negative value of the resistance is due to the control system suppressing the mechanical damping of the passive structure in order to create a fully reactive periodic system in which a perfect theoretical band gap would exist.

As an alternative, by choosing a criterion based on the maximization of the electrical damping it is possible to modify the dynamic properties of the plate by increasing the energy absorption properties of the active interface. Optimization of the electric synthetic impedance parameters for this criterion leads to design of an absorbing metacomposite able to reduce the vibratory energy flowing through it by increasing the power dissipated within the electric circuit. This optimal case requires a negative capacitance and a positive and larger frequency-dependent resistance as compared to the reactive interface criterion described above.

However, the required electrical impedances for these two circuit optimization approaches cannot be readily reproduced experimentally because it is not possible to exactly recreate the optimal frequency dependent resistance and capacitance over the whole frequency range of interest.

For the work presented here, the layout of the actual circuit implemented for control of the patches is presented in Figure 2. This circuit contains a number of passive components, including the resistances $R, R_{2}, R_{3}, R_{4}$ and the capacitance $C_{2}$, as well as an active component, specifically, an operational amplifier that is essential for the circuit to reproduce the desired negative capacitance behavior. This specific circuit layout was chosen as opposed to others (Fleming et al. (2000)) because of its simplicity and its effectiveness in the frequency range of interest. The impedance of the circuit in Figure 2 is determined by the formula

$$
Z_{e q}(\omega)=R_{e q}+\frac{1}{i \omega C_{e q}}=R-\frac{R_{3} R_{2}}{R_{4}\left(1+i \omega R_{2} C_{2}\right)} .
$$

Figure 2 depicts the frequency-dependent behavior of the actual impedance produced by the real-life circuit; in the upper part of Figure 2 the equivalent resistance $R_{e q}$ is compared to the ideal resistance $R$ and at the bottom the equivalent capacitance $C_{e q}$ is compared to the ideal capacitance $C$. Both terms vary as a function of the frequency; in particular the resistance at low frequency presents a negative value that can introduce some instability which can degrade the overall performance of the control system and it is compensated by a positive input resistance $R$. For relatively high frequency values, the equivalent resistance $R_{e q}$ and the equivalent capacitance $C_{e q}$ are modified according to the following formulae

$$
R_{e q}=R, \quad C_{e q}=C_{n e g}=-\frac{R_{4}}{R_{3}} C_{2} .
$$

The negative impedance control technique requires tuning of the synthetic capacitance around the capacitance value of the piezoelectric ceramic at free stress condition (Livet et al. (2011)); this value is intrinsic to the electromechanical coupling and depends upon the material properties, the geometry of the patches and the plate stiffness. The intrinsic capacitance of the piezoelectric patch can be estimated by measurement using a capacitance meter when the specimen is free of external forces. In the present case the measured nominal patch capacitance is $50 \mathrm{nF}$. The actual circuit was tuned by varying the ratio $R_{4} / R_{3}$ in small increments from high negative values of the synthetic capacitance to a value very close to the targeted intrinsic capacitance value. When the external circuit approaches the set-point the system tends to be unstable and an increase of output voltage is observed (Livet et al. (2011)). If the output voltage reaches the limits of the op-amp's power 


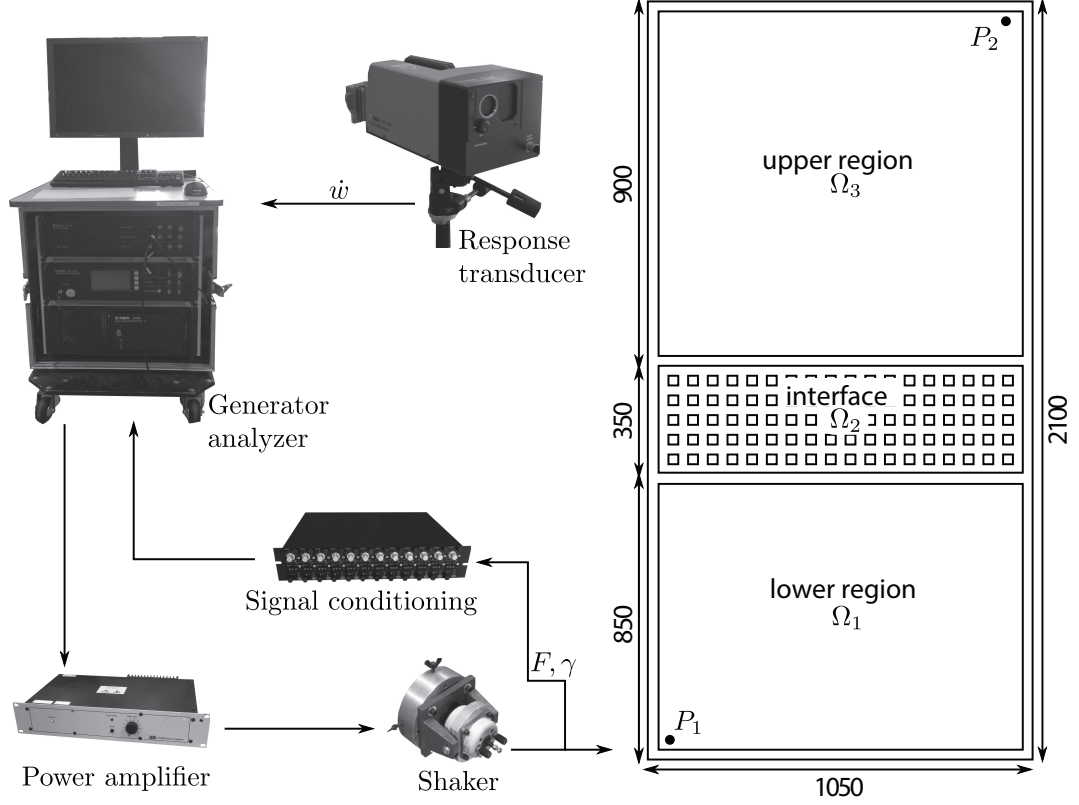

Figure 3: Layout of FRF measurement system. $P_{1}$ is the force input position, while $P_{2}$ used for a representative single-point transfer mobility measurement.

supply voltage then the operational amplifier saturates and its output clips. In order to mitigate this effect, the circuit was modified by adding a complementary circuit able to detect saturation of the output. Basically, a comparator was introduced to monitor the op-amp output in order to identify these voltage overloads.

For the work at hand, two configurations of the of circuit have been implemented in order to program two different vibroacoustic behaviors:

- Case 1 corresponding to a lightly-damped, reflective configuration. It is obtained by tuning the negative capacitance $C_{n e g}$ very close to the instability point of the piezoelectric $\left(C_{n e g}=\right.$ $-52 n F)$. The input resistance $R$ is set to a small positive value able to reproduce the desired behavior (wave reflection) and still ensuring the stability of the control system $(R=40 \Omega)$.

- Case 2 corresponding to an absorbing configuration. It is obtained by tuning the negative capacitance $C_{n e g}$ with the same value of Case $1\left(C_{n e g}=-52 n F\right)$. The energy absorption enhancement is then obtained by imposing a larger input resistance $(R=400 \Omega)$.

\subsection{Experimental set-up}

As noted previously, the structure of interest, shown in Figure 1, consists of an aluminum plate equipped with 75 piezoelectric actuators connected to external circuits and arranged so as to form a periodic array of $5 \times 15$ units. Standard epoxy glue was used to bond the piezoelectric ceramics to the top surface of the plate. Elastic waves are excited by a shaker located in the lower corner of the plate, $850 \mathrm{~mm}$ away from the active interface at the point labeled $P_{1}$ in Figure 3 . The input transducer is driven by an input signal consisting of a random broad-band excitation and amplified 


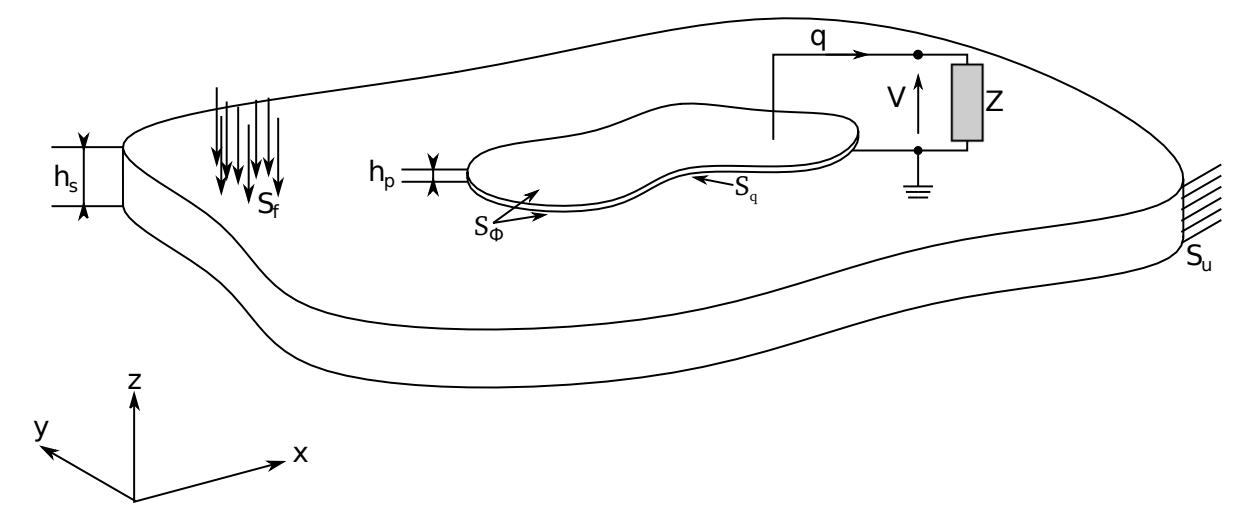

Figure 4: Plate with arbitrarily shaped piezoelectric ceramic shunted to an electric impedance.

by an LDS power amplifier. The response of the structure to the input disturbance and action of the active interface was measured with a scanning laser vibrometer (Polytec PSV-400).

Measurements were conducted over a grid of $37 \times 81$ scan points with a spatial resolution of $20 \mathrm{~mm}$ in the horizontal and vertical directions. At each grid point, the laser measured the time history of the out-of-plane velocity.

\section{Numerical Model}

\subsection{Mathematical Formulation}

Consider the flexible piezoelectric structure depicted in Figure 4 occupying the volume $V_{s}$ and subjected to a prescribed displacement $\bar{u}$ on a part $S_{u}$ and to surface force distribution $f$ on the complementary part $S_{f}$ of its external boundary. Let $n$ be the unit normal external to $V_{s}$. The electric boundary conditions are defined by a prescribed surface charge $\bar{q}$ on $S_{q}$ and an electric potential $\bar{\phi}$ on the remaining part $S_{\phi}$. The local set of equilibrium equations describing the mechanical and the electrical domain are given by

$$
\begin{aligned}
\rho \ddot{u}-\nabla \cdot \sigma & =f \\
\nabla \cdot D & =0
\end{aligned}
$$

where $u$ is the displacement field, $\sigma$ is the symmetric Cauchy stress tensor, $D$ denotes the electric displacement vector components and $\rho$ is the mass density of the structure. The differential problem is defined once the boundary conditions are defined

$$
\begin{array}{ll}
\sigma \cdot n=T & \text { on } S_{f} \\
u=\bar{u} & \text { on } S_{u} \\
D \cdot n=-\bar{q} & \text { on } S_{q} \\
\phi=\bar{\phi} & \text { on } S_{\phi}
\end{array}
$$

where $T$ indicates the component of the stress tensor projected along the outpointing normal, $\bar{u}$ the imposed displacement, $\bar{q}$ the imposed charge and $\bar{\phi}$ the imposed voltage.

The stress tensor and electric displacement are related to the linear strain tensor $\varepsilon$ and electric 
field $E$ through the constitutive laws for a piezoelectric material (Royer and Dieulesaint (2000))

$$
\begin{aligned}
\sigma & =C_{E} \varepsilon-e^{T} E \\
D & =e \varepsilon+\epsilon_{S} E
\end{aligned}
$$

where $C_{E}, e, \epsilon_{S}$ denote the mechanical stiffness matrix evaluated at constant electric field, the piezoelectric stress coupling matrix and the permittivity matrix at constant strain, respectively.

The strain tensor is calculated as

$$
\varepsilon=\nabla_{s} u=\frac{1}{2}\left(\nabla u+\nabla^{T} u\right)
$$

while the electric field and the voltage are related through the simple formula

$$
E=-\nabla \phi
$$

Within a finite element framework, the field variables $u$ and $\phi$ are related to nodal degrees of freedom $d$ and $V$ through a proper selection of shape functions $N_{u}$ and $N_{\phi}$

$$
u=N_{u} d \quad \phi=N_{\phi} V .
$$

By assuming an harmonic motion at frequency $\omega$ for each variable the following electro-elastic system of equations is obtained

$$
\begin{aligned}
K_{u u} \hat{d}+K_{u \phi} \hat{V}-\omega^{2} M_{u u} \hat{d} & =\hat{f} \\
K_{\phi u} \hat{d}+K_{\phi \phi} \hat{V} & =\hat{q}
\end{aligned}
$$

the symbol . over each physical quantity denotes its complex amplitude in the frequency domain. The combination of the host structure and the piezoelectric patches is modeled using 4-node Kirchhoff plate elements (Hughes (2000)), the electric field is assumed to be constant on the surface of each piezoelectric patch and the electric potential is assumed to vary linearly through the thickness. Under these assumptions, the electrical equation can be reduced to the form

$$
K_{\phi u} \hat{d}+K_{\phi \phi} \hat{V}_{p}=\hat{q} .
$$

Application of the shunting circuit to the electrodes of the piezoelectric patch allows condensing out equations (9) to obtain

$$
\left(K_{u u}-\omega^{2} M_{u u}+S_{u u}\right) \hat{d}=\hat{f}
$$

where $S_{u u}$ is the shunting matrix describing the effect of the shunting circuits on the dynamic behavior of the structure. It is given by

$$
S_{u u}=j \omega K_{u \phi}\left(j \omega K_{\phi \phi}-K_{Z}\right)^{-1} K_{\phi u}
$$

where $K_{\phi \phi}$ is the matrix containing the terms related to the intrinsic capacitance $C_{p}$ and $K_{Z}$ is the matrix containing the terms related to the impedance $Z$ of the shunting circuit in Figure 4 . The impedance of each piezoelectric ceramic establishes a relation between the voltage $\hat{V}$ and the flowing charge $\hat{q}$ according to the following equation

$$
\hat{V}=Z j \omega \hat{q} .
$$

It is clear that the shunting matrix can affect the inertial, the damping or the stiffness properties of the structure depending on the characteristic of the piezoelectric patch and the shunting circuit parameters contained in the impedance $Z$. 


\subsection{Energy Balance for Harmonically Excited Systems}

To better understand the underlying physics of this control system an energy balance analysis including time-varying quantities such as velocities and forces has been conducted (Bobrovnitskii (2004)). Equation (11) represents a linear n-DOF mechanical system vibrating harmonically under the action of an external force.

The main goal of this section is to recall some equations relating various energy characteristics such as the power flow, the kinetic energy and others. Under an harmonic excitation each of these quantities can be represented as the sum of three harmonically varying components, as an example let us consider the power flow

$$
\mathcal{P}(t)=F(t) \cdot v(t)=P_{0}+P_{c} \cos 2 \omega t+P_{s} \sin 2 \omega t .
$$

where $F(t)$ and $v(t)$ are the time histories of the force and the velocity associated to a point belonging to the domain. The three components in equation (14) are independent in the sense that they are orthogonal in the time interval $2 \pi / \omega$.

The terms $P_{0}, P_{c}$ and $P_{s}$ can be easily estimated using the complex numbers formalism, for example the alternating components of $\mathcal{P}$ in the frequency domain is simply the product of the complex amplitude of the velocity and the force

$$
P=v^{T} F=P_{c}+j P_{s} .
$$

likewise the continuous components are calculated as the product of the force and the conjugate of the velocity

$$
P^{*}=v^{* T} F=P_{0}+j P_{q} .
$$

The term $P_{q}$ is not related to any significant physical quantity but rather represents the part of the velocity vector in quadrature with the external force.

Other quadratic quantities such as the kinetic and the potential energy are represented in a similar manner. However, unlike the power flow, they all have zero reacting component. For example we can consider the kinetic energy

$$
\begin{aligned}
T & =\frac{1}{4} v^{T} M v=T_{c}+j T_{s} \\
T^{*} & =\frac{1}{4} v^{* T} M v=T_{0}
\end{aligned}
$$

or the potential energy

$$
\begin{aligned}
W & =\frac{1}{4} u^{T} K u=W_{c}+j W_{s} \\
W^{*} & =\frac{1}{4} u^{* T} K u=W_{0}
\end{aligned}
$$

the sum of the kinetic energy $T_{0}$ and the potential energy $W_{0}$ determines the total energy $E_{0}$ of the system

$$
E_{0}=W_{0}+T_{0}
$$

All these quantities allows one to qualify the performances of the control system. For example by calculating the active power flow $P_{0}$ and the total energy $E_{0}$ it is possible to estimate the loss factor $\eta$ of the structure (Bobrovnitskii (2004)) by simply calculating

$$
\eta=\frac{P_{0}}{\omega E_{0}} .
$$


Another important quantity able to quantify the amount of kinetic energy confined in a specific portion of the plate is kinetic energy ratio $\tau$

$$
\tau=\frac{T_{0}^{i}}{T_{0}^{t o t}}
$$

where $T_{0}^{\text {tot }}$ represents the sum of three constituting regions of plate plate, namely the lower and the upper region and the interface (Figure 3).

\section{Results}

\subsection{Harmonic Response of the Metacomposite}

The host structure is a rectangular plate with geometrical and mechanical properties summarized in Figure 3. A periodic lay-out of $15 \times 5$ piezoelectric patches is placed on the top surface of the plate $850 \mathrm{~mm}$ away from the lower side.

The plate is excited by a point force located in the lower left corner of the plate (point $P_{1}$ ), the response is evaluated in terms of out-of-plane velocity calculated at point $P_{2}$ and its derived quantities such as the loss factor and the kinetic energy ratio. The FE mesh features $46 \times 70$ rectangular plate elements.

The present model has been validated by comparing the calculated and the measured natural frequencies of the non controlled plate equipped with the piezoelectric ceramics which are connected to the external electrical circuits in absence of power. Table 2 summarizes the set of natural frequencies used for the comparison and shows that the relative error in less than $5 \%$ for all the values.

In Figure 5 the calculated and the measured loss factors are compared in three different conditions: open circuit, negative capacitance control strategy with $R=40 \Omega$ and $R=400 \Omega$. In Figure 5 the lines are associated to the experimental measurements while the bars are associated to the calculated solutions. The blue items represent the baseline solution, by comparison it is clear that the calculated solution underestimate the total amount of dissipated energy. For example is not easy to include in the considered model the dissipation mechanism associated to the friction of the electric wires with the plate's surface (Figure 1). Another neglected source of dissipation is the external electric circuit, in the theoretical model each circuit is assumed to be open but this is not the case since each circuit is still connected to the external circuit in absence of power. Under these conditions the circuit is not able to reproduce the negative capacitance effect but it is reasonable to assume that each circuit can still add an extra amount of damping due to the current flowing within the circuit.

The different behavior observed at low frequencies is also motivated by the approach used for estimating the loss factor. In the numerical model each energy related quantities are easily available,

\begin{tabular}{llllllllll}
\hline & $f_{1}$ & $f_{2}$ & $f_{3}$ & $f_{4}$ & $f_{5}$ & $f_{6}$ & $f_{7}$ & $f_{8}$ & $f_{9}$ \\
\hline Ref & 3.65 & 4.69 & 9.62 & 9.97 & 14.79 & 17.34 & 17.59 & 19.78 & 23.91 \\
Exp & - & 4.62 & - & 9.81 & - & - & - & 19.90 & 23.40 \\
Err $\%$ & - & 1.50 & - & -1.90 & - & - & - & -0.60 & 2.13 \\
\hline
\end{tabular}

Table 2: Comparison of the experimental and numerical plate's natural frequencies. 


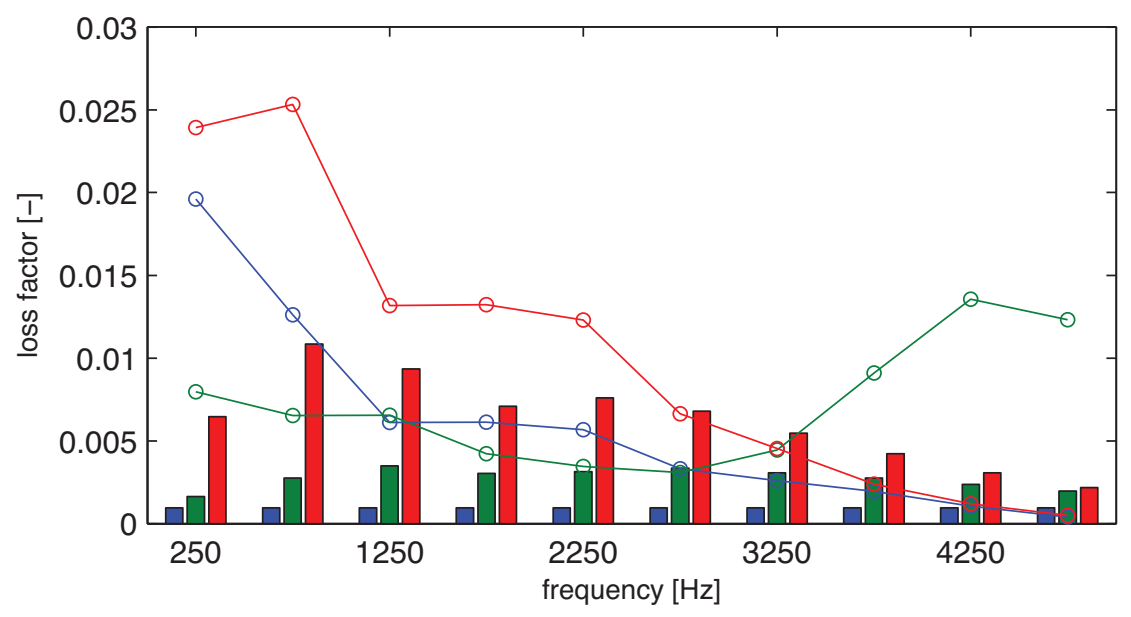

Figure 5: Loss factor of the smart plate, the lines represent the experimental data and the bars the calculated solutions. The color code indicates: the uncontrolled plate (blue), controlled plate with $R=40 \Omega$ (green) and $R=400 \Omega$ (red).

for example, the calculation of the potential energy $W_{0}$ is obtained once the stiffness matrix is defined. Thus, the total energy $E_{0}$ is calculated taking into account the contribution due to kinetic energy $T_{0}$ and the potential energy $W_{0}$. Experimentally the estimation the total energy $E_{0}$ is more difficult to obtain and a different approach is used: the total energy $E_{0}$ is calculated as the double of the kinetic energy $T_{0}$. However this assumption only holds in presence of high modal density. This restraint implies that reliable results can be obtained only for high frequency values. Moreover the estimation of the kinetic energy is affected by additional errors due to the numerical integration of the velocity field measured at the meshing points. Despite this limitations the calculation of the loss factor still represents a good indicator of the damping capabilities of the control system over the whole frequency band, low frequencies included. It should point out that the calculated loss factor represent a global information that not entirely reflect the absorbing capabilities of the considered control system. The calculation of the loss factor consider the energy contained in the whole plate included the region of the plate not directly controlled by the piezoelectric shunts. To better understand this point an additional analysis has been initiated. The aim of this study is to locally characterize the physical properties of the smart plate.

In Figure 6 the calculated and measured kinetic energy ratio is compared in three different conditions: open circuit, negative capacitance control strategy with $R=40 \Omega$ and $R=400 \Omega$. The results are collected in three different diagrams representing respectively the ratio of kinetic energy confined in the upper portion of the plate, within the plate and in the lower portion of the plate. If we consider the part of the plate under the smart interface larger values of the kinetic energy ratio are associated to an increase of kinetic energy in the considered area. Under these conditions the vibrating field is modified, the smart interface is capable to confine the kinetic energy in a specific portion of the plate. The energy confined in the lower portion of the plate increases from 1250 to $3250 \mathrm{~Hz}$ for the absorbing configuration and then is shifted toward higher frequencies decreasing the input resistance. Beyond $2500 \mathrm{~Hz}$ the reflecting configuration provide better performances; the active interface acts like an electro-mechanical filter that prevents the occurrence of the standingwaves. Considering the top of Figure 6 similar conclusions can be drawn, in this case, however, 
smaller values of the kinetic energy ratio correspond to the energy confinement effect. The obtained results are in good agreement with the experimental measurements even if the theoretical results constantly overestimate the confined energy. For higher frequencies the measurements and the calculated response are not in perfect agreement. This mismatch is partly motivated by the degradation of the the coherence function observed at higher frequency values.

The relevance of the mathematical model is finally illustrated by comparing the velocity field of the controlled plate presented in Figure 7 for different excitation frequencies. At $25 \mathrm{~Hz}$ the response associated to the open circuit configuration is correctly captured by the mathematical model, the ODS are almost identical. The same trend is observed considering a negative capacitance circuit with a resistance of $R=400 \Omega$. However, with the negative capacitance circuit set with a resistance of $R=40 \Omega$ the results are less satisfactory since it is not possible to reproduce the same ODS. At $1680 \mathrm{~Hz}$ the measured and the theoretical responses are again comparable in all cases, here the comparison becomes more difficult since the spatial resolution is not sufficient to correctly describe the actual vibrating field, despite this limitation the similar repartition of the energy within the plate provides indication about the quality of the mathematical model. 

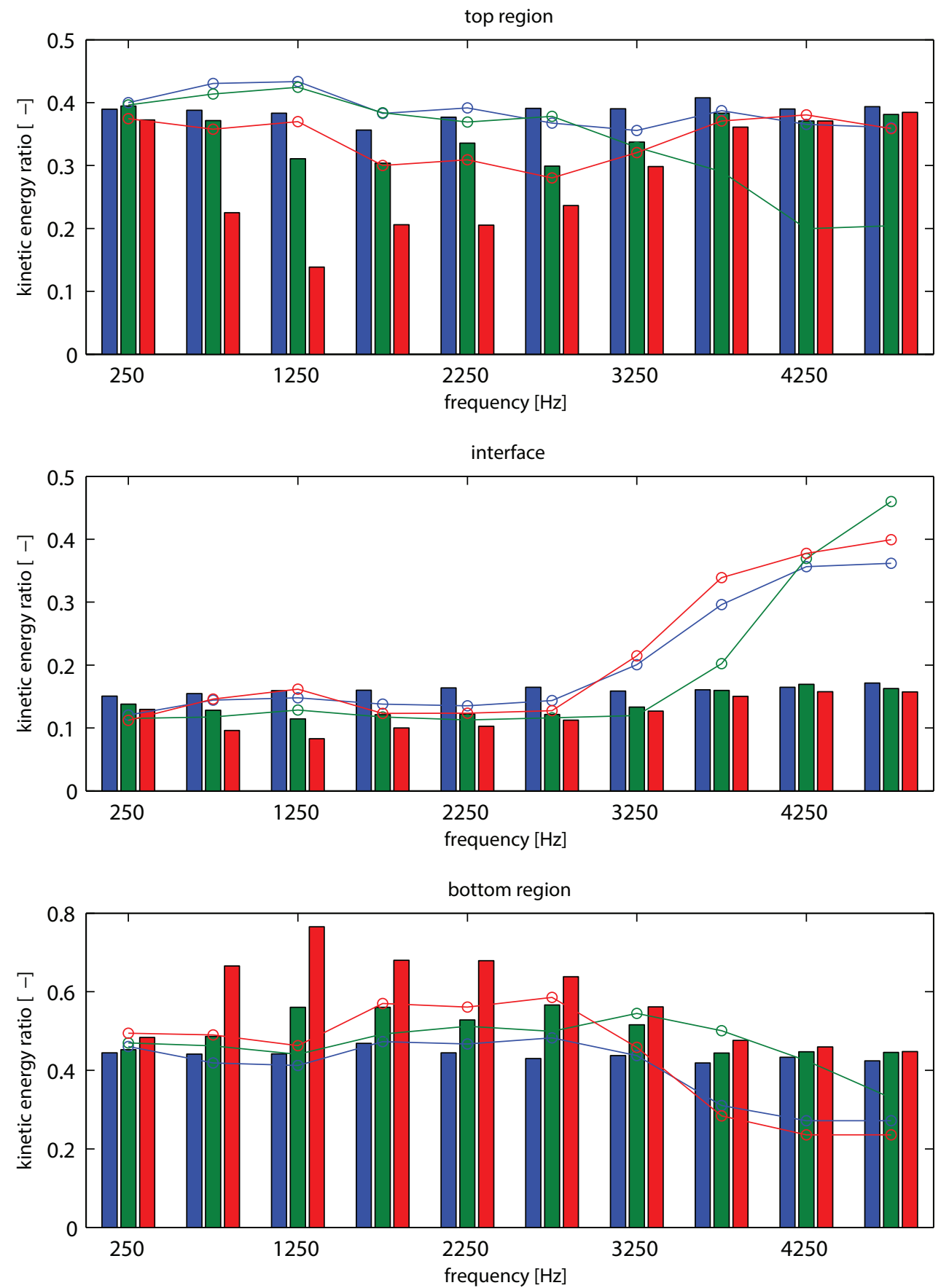

Figure 6: Kinetic energy distribution of the smart plate, the lines represent the experimental data and the bars the calculated solutions. The color code indicates: the uncontrolled plate (blue), controlled plate with $R=40 \Omega$ (green) and $R=400 \Omega$ (red). 
reference (th)

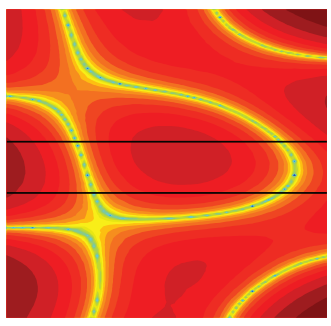

reference (exp)

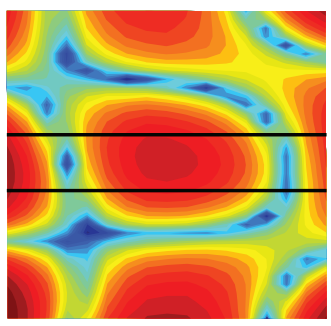

reference (th)

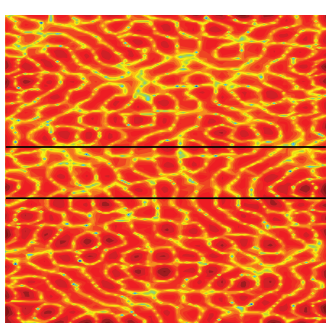

reference (exp)

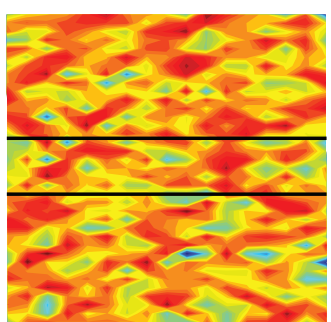

RC: 40 ohm (th)

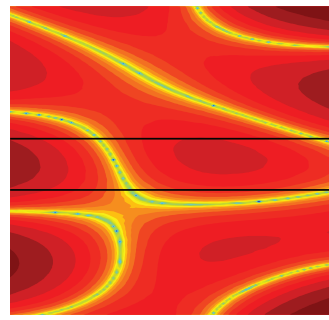

RC: 40 ohm (exp)

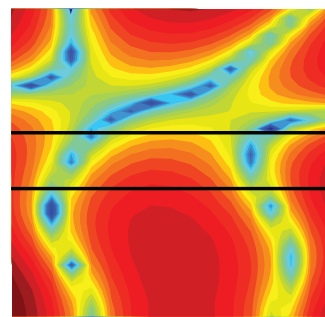

RC: 40 ohm (th)

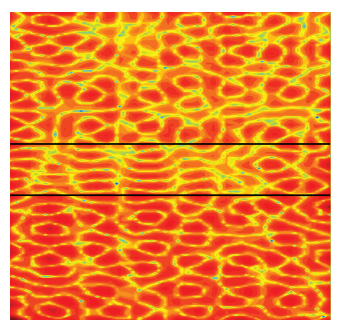

RC: 40 ohm (exp)

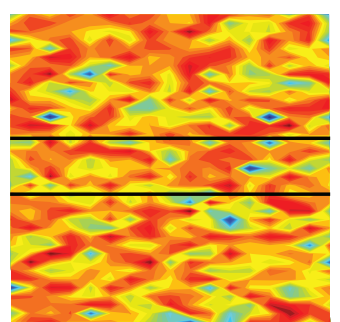

$\mathrm{RC}: 400$ ohm (th)

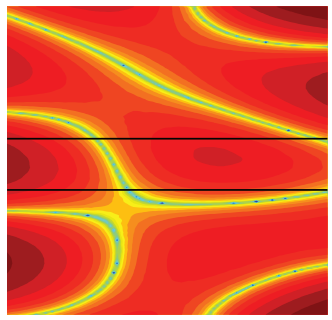

RC: 400 ohm (exp)

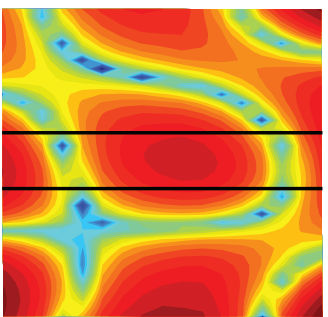

RC: 400 ohm (th)

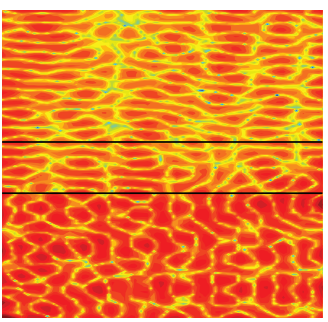

RC: 400 ohm (exp)

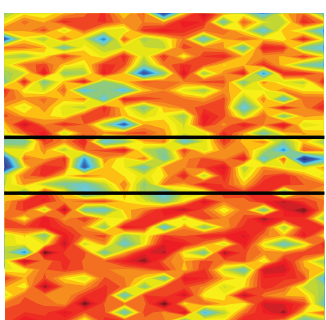

Figure 7: Two dimensional velocity field comparison (theoretical vs experimental data) of the smart plate: uncontrolled plate (left), controlled plate with $R=40 \Omega$ (center) and $R=400 \Omega$ (right). Two excitation frequency have been considered: $25 \mathrm{~Hz}$ (top) and $1680 \mathrm{~Hz}$ (bottom). 

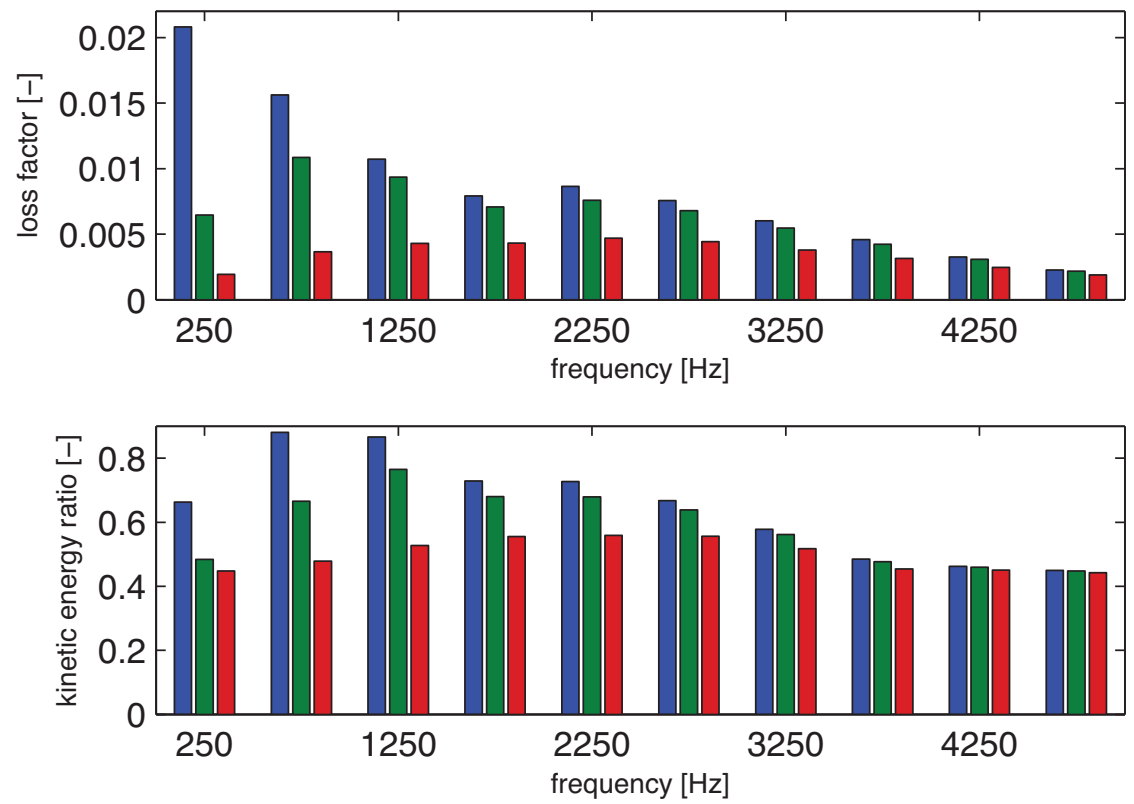

Figure 8: Loss factor and lower region's kinetic energy distribution of the smart plate for different value of negative capacitance. The blue bars indicates the controlled plate $C_{n e g}=-51.3 n F$; the green bars the controlled plate $C_{n e g}=-55.7 n F$ and red bars the controlled plate $C_{n e g}=-59$ $n F$. In all cases a resistance of $R=400 \Omega$ has been chosen.

\subsection{The Effect of the Circuit's Parameters}

The performances of the control system can be improved by properly tuning the external circuit. In order to highlight this dependency a parametric analysis has been performed considering a variation of the negative capacitance.

In Figure 8 the loss factor of the controlled structure is compared to the reference solution for three different values of negative capacitance. The blue bars are associated to a negative capacitance of $51.3 n F$ while the green and the red bars represent a negative capacitance of 55.7 and $59 n F$ respectively.

As expected the performances of the system degrade as one move away from the point corresponding to the opposite of the intrinsic capacitance $C_{p}$. An interesting feature is the fact that larger attenuation is obtained for $C_{n e g}=51.3 \mathrm{nF}$ over a narrower frequency band, namely $0-1000 \mathrm{~Hz}$ whereas for a bigger value of negative capacitance the overall performances are slightly reduced but broadened up to $5000 \mathrm{~Hz}$. The filtering properties of the smart interface are enhanced introducing a negative capacitance control system. In particular with negative capacitance values closed to $C_{p}$ the system is able to increase this indicator up to 0.8 in the $1000 \mathrm{~Hz}$ frequency band (Figure 8). The red bar representing the largest considered value of negative capacitance still shows acceptable performances, this fact can be used as illustration of the robustness of the proposed system.

The circuit's model can be further refined by considering the actual impedance as presented in equation (1). The layout of the actual circuit includes an operational amplifier employed as a gyrator. This specific circuit architecture originates a negative resistance at low frequencies that determine an energy increase detrimental for the system's stability as shown in the equation of the 

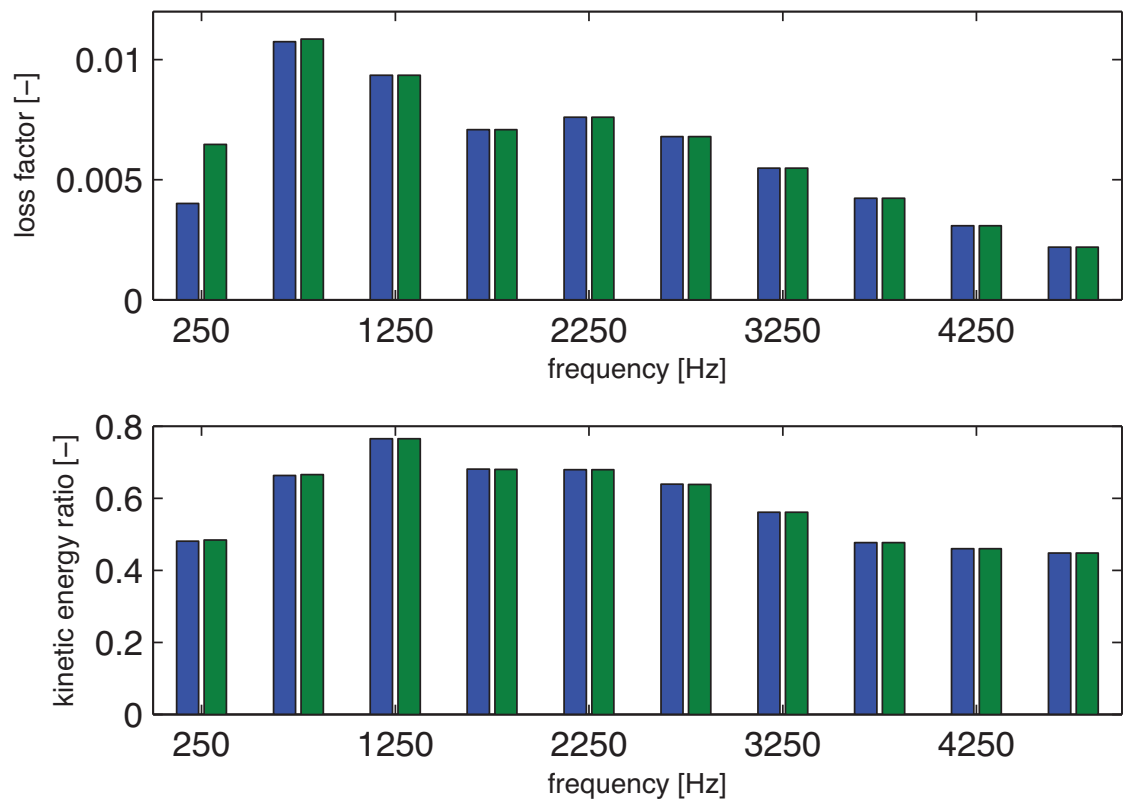

Figure 9: Loss factor and lower region's kinetic energy distribution of the smart plate for different circuit layout. The blue bars indicate the controlled plate with real-life circuit and the green bars the ideal circuit with an input resistance of $R=400 \Omega$. The parameters of the real-life circuit have been chosen in order to reproduce the same capacitance at higher frequencies.

real-life impedance.

This effect is clearly observed in Figure 9 where the experimental loss factor in the $250 \mathrm{~Hz}$ frequency band assumes the value of 0.025 , it increases up to almost 0.03 and then decreases when the excitation frequency is increased. This trend is better observed for the real life circuit represented by the blue bars, in this case the loss factor is decreased at low frequencies. At higher frequency values no differences between the two mathematical models are observed. This last observation suggests that in most of the cases the former approach can be used without loss of reliability. We should point out that both approaches lack of some prediction capabilities due to the fact that is not easy to take into account each aspect of the real-life circuit in particular the actual behavior of the operational amplifier and the contribution of the auxiliary circuit introduced in order to detect the occurring instability (LED circuit).

Regarding the kinetic energy distribution (Figure 9) the two models give the same results showing that the enhanced circuit model has an effect on the active part of the impedance leaving unchanged the reactive ones. 

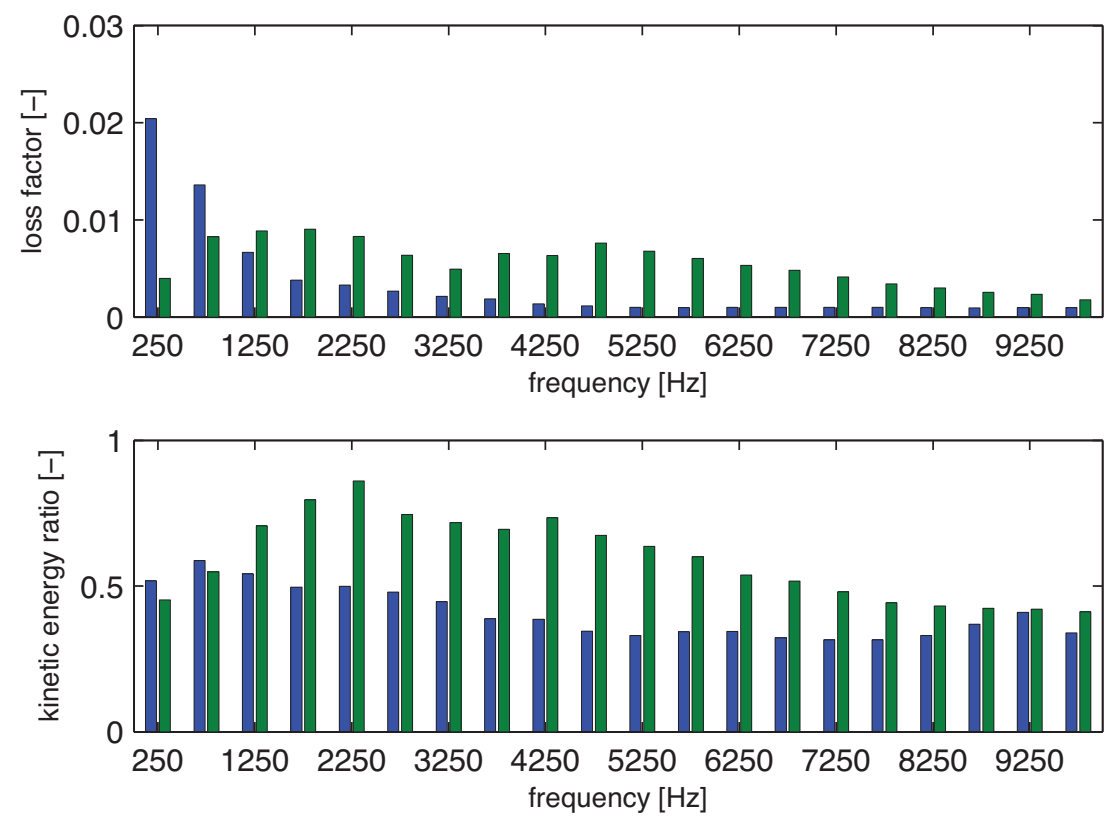

Figure 10: Loss factor and lower region's kinetic energy distribution of the smart plate for different interface topologies. The blue bars indicate the controlled plate with type B interface and the green bars the controlled plate with type $\mathrm{C}$ interface. In both cases an input resistance of $R=400$ $\Omega$ has been selected.

\subsection{Smart Interface Acting Like a Robust Frequency Filter}

The section's main objective is to show how the control system acts as a filter capable of suppress vibrations in a specific frequency band. To do so the topology of the smart interface has been analyzed, by variating the geometry of the piezoelectric actuators or the layout of the smart interface is possible to modify the behavior of the control system.

These mechanisms have been analyzed by considering two different interface's layouts presenting an array of $15 \times 5$ (interface type B) and $20 \times 7$ (interface type C) piezoelectric actuators respectively and for each of which different ceramics' dimensions have been considered, see Table 1.

In Figure 10 the structure's loss factor is compared for the two different interface layout. The blue bar associated to the configuration $15 \times 5$ shows higher loss factor values at very low frequencies, in the $250 \mathrm{~Hz}$ frequency band the averaged loss factor exceeds the value of 0.02 for higher values a deterioration in performance is observed in particular beyond $1000 \mathrm{~Hz}$ the $15 \times 5$ configuration appears less effective than configuration $20 \times 7$ (green bar). Configuration $20 \times 7$ provides good performances from 1000 to $10000 \mathrm{~Hz}$. Moreover, respect to configuration $15 \times 5$ the value of the loss factor decrease less dramatically from one band to another, for instance a value of almost 0.01 is preserved over a frequency range of almost $5000 \mathrm{~Hz}$. According to these results it is clear that the dimensions of the smart interface have a large impact on the performance of the control system. As a rule of thumb the length of the piezoelectric actuator should respect the following condition

$$
L<\frac{\lambda^{*}}{2}
$$


where $\lambda^{*}$ represent the wavelength of the targeted mode. For example examining the dispersion relation of the $A_{0}$ mode of the bare plate at $10000 \mathrm{~Hz}$ one obtains a wavenumber $k^{*}=120 \mathrm{rad} / \mathrm{m}$ and a wavelength $\lambda^{*}=5.2 \mathrm{~cm}$, this means that the maximum length of the ceramic will be less than $2.6 \mathrm{~cm}$. This result is in good agreement with the calculated response. A $3 \mathrm{~cm}$ long ceramic is not able to exert any appreciable control in this specific frequency band.

Similar considerations can be done about the averaged kinetic energy distribution within the plate. Configuration $15 \times 5$ represented by blue bars in Figure 10 shows that the energy is evenly spread in the bottom part of the plate, there is not preferred frequency band, even if at very low frequencies the active interface seems to better filter the vibrating field increasing the ratio up to 0.6. Interface type B represented by green bars in Figure 10 behaves in a better way, in the $250-8250 \mathrm{~Hz}$ the energy ratio is increased from 0.5 to 0.8 .

A certain control ability is exercised over a specific frequency band once the dimensions of the periodic array and the circuits' parameters have been selected. However, in real life applications it is not easy to exactly determine the most appropriate set of parameters because of the uncertainties due to: the electromechanical coupling; the dispersion of the mechanical properties of the piezoelectric ceramic and the electrical components for which a nominal tolerance is provided by the manufacturer itself.

With these premises it is not possible to build a perfect periodic lattice of piezoelectric actuators but rather a network of co-localized actuator driven by an independent negative capacitance circuit.

These sources of uncertainty are taken into account in the mathematical model by selecting for each external circuit a negative capacitance that varies according to an uniform distribution between 51 and $53 \mathrm{nF}$.

At the top of Figure 11 the loss factor is compared considering a set of circuits having an input resistance of $400 \Omega$ and a constant value of negative capacitance and a group of circuits having the same value of resistance and a variable value of negative capacitance. As expected a performance modification is observed, for example around $1000 \mathrm{~Hz}$ the loss factor associated to the second configuration (green bars) varies, respect to the ideal case with $C_{n e g}=51.3 n F$ and $R=400 \Omega$ (blue bars), from 0.0011 to 0.0010 . The kinetic energy ratio associated to the lower part of the plate is less perturbed by the destruction of the array's periodicity (bottom of Figure 11).

The smart interface is also not very sensitive to the type of structure to control, no prior knowledge of the system's modes is required. To point out this feature in Figure 12 the mechanical response of the system measured in a point located in the upper region of plate has been considered (point $P_{2}$ ). The comparison between the uncontrolled response (blue line) and the controlled one (green line) shows that control system is able to considerably reduce the system's response. At the top of Figure 12 the response of the plate has been calculated imposing a thickness of $3 \mathrm{~mm}$, at the bottom of Figure 12 the thickness of the plate has been increased up to $9 \mathrm{~mm}$. In both cases the mechanical response of the plate is attenuated in the whole frequency band. 

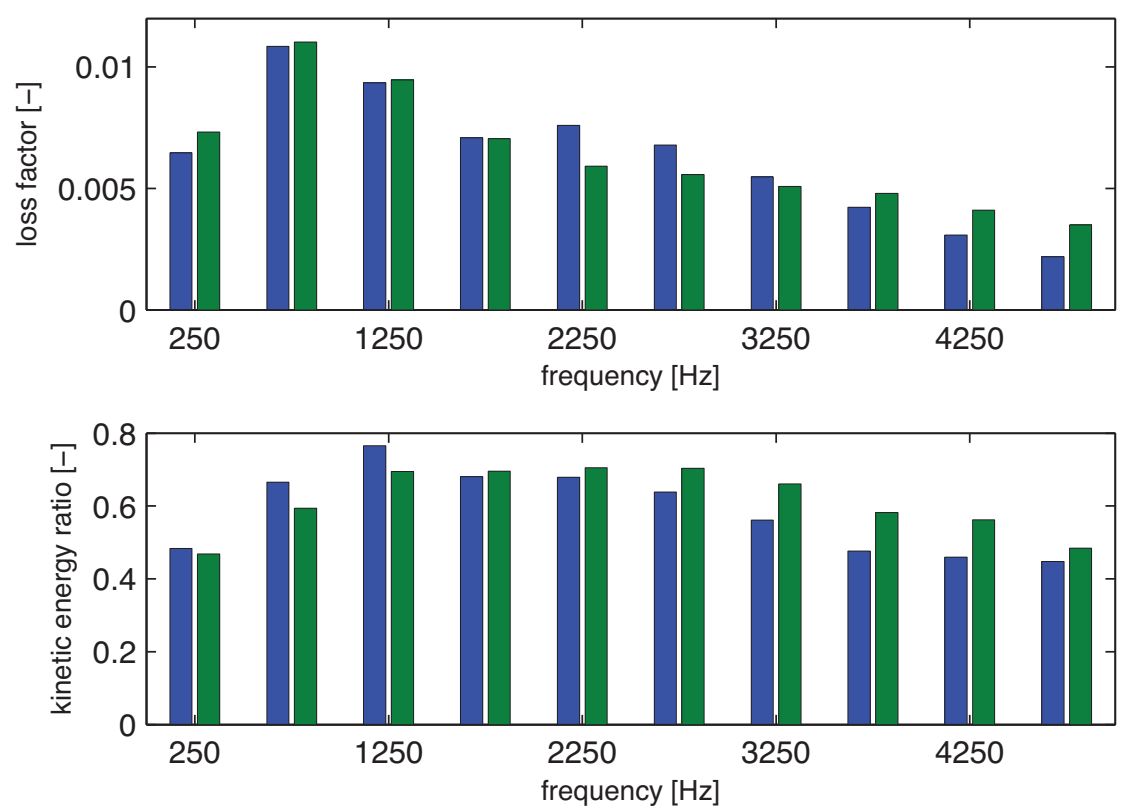

Figure 11: Loss factor and lower region's kinetic energy distribution of the smart plate with a loss of periodicity. The blue bars indicate the controlled plate with a periodic lattice of piezoelectric actuators; the green line the controlled plate with randomly distributed value of negative capacitance. For both circuits $R=400 \Omega$.
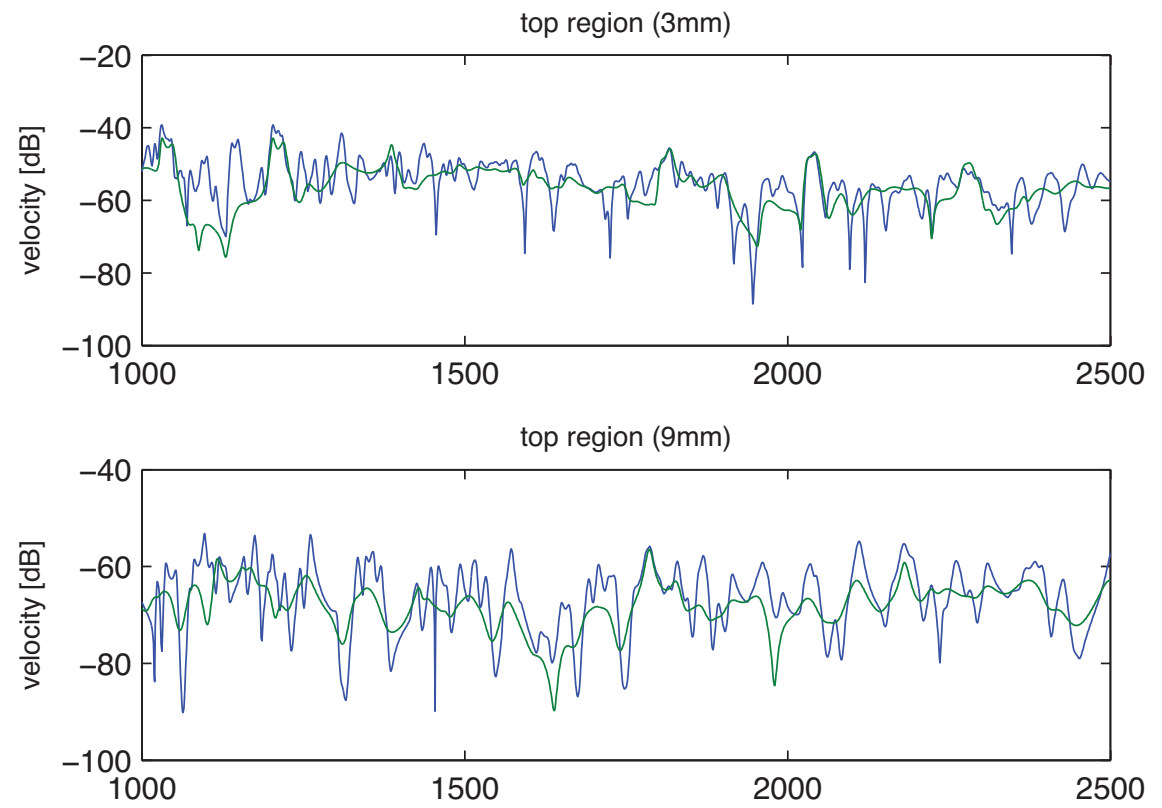

Figure 12: Frequency response function of the periodic plate (interface type A) for two different circuit configuration: uncontrolled (blue line); controlled (green line). Two different plate's thicknesses are considered: $3 \mathrm{~mm}$ (top); $9 \mathrm{~mm}$ (bottom). 

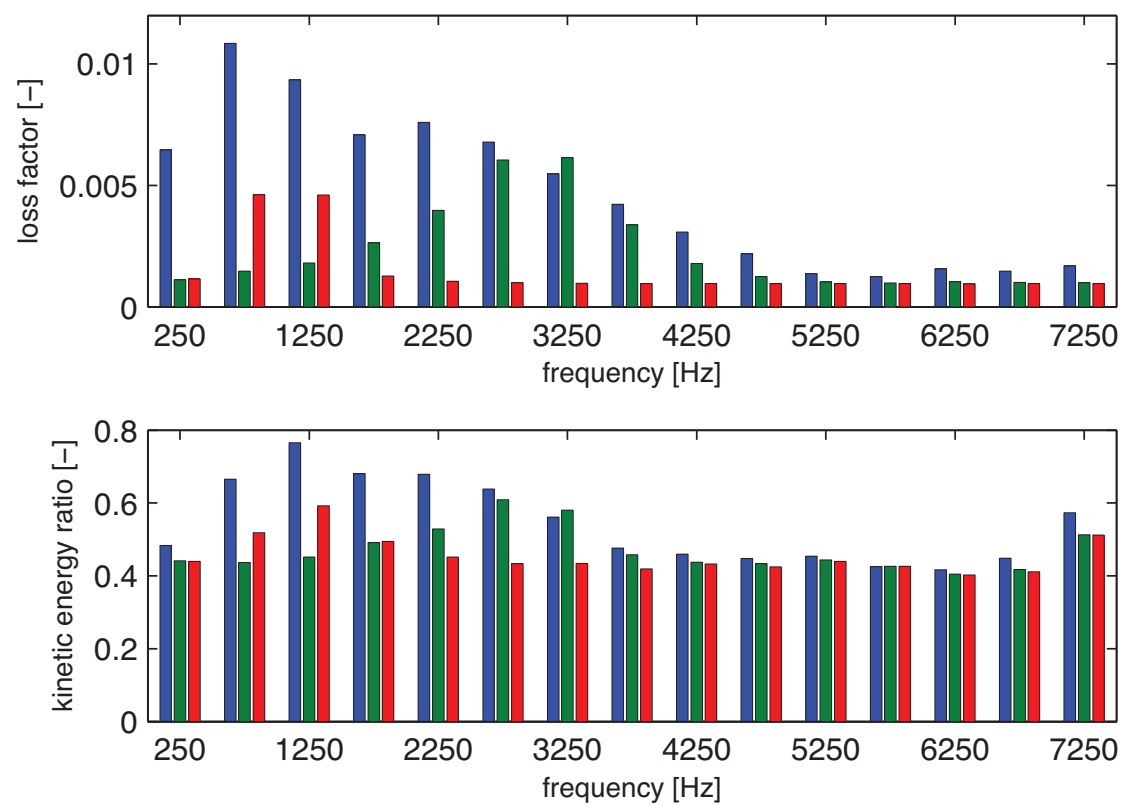

Figure 13: Loss factor and lower region's kinetic energy distribution of the smart plate controlled by means of resonant and negative capacitance circuits. The blue bars represent the negative capacitance circuit with $C_{n e g}=-52 n F$ and $R=400 \Omega$; the red bars the resonant circuit with $f_{\text {tun }}=1000 \mathrm{~Hz}$ and $R=400 \Omega$; the green bars the resonant circuit with $f_{\text {tun }}=3000 \mathrm{~Hz}$ and $R=400 \Omega$.

\subsection{Negative Capacitance vs. Resonant Circuits}

In this section two control strategies based on negative capacitance circuits and resonant circuits are compared. To do so a $15 \times 5$ array of piezoelectric patches is considered, for a sake of convenience the geometric properties of the smart interface are identical to the layout described in Table 1 (interface type A).

For the resonant circuit the controlling effect is obtained by correctly tuning the external circuit composed by a resistance $R$ in series with an inductance $L$. Two different tuning frequencies are arbitrarily selected at $f_{\text {tun }}=1000$ and $f_{\text {tun }}=3000 \mathrm{~Hz}$. The negative capacitance circuit is set imposing a negative capacitance value of $51.15 n F$. For both layouts an input resistance $R$ of 400 $\Omega$ is selected.

The value of the inductance $L$ is chosen in order to tune the shunt circuit at the desired resonant frequency according to

$$
L=\frac{1}{\omega_{\text {tun }}^{2} C_{p}}
$$

where $C_{p}$ is the inherent capacitance of the piezoelectric patch and $\omega_{\text {tun }}=2 \pi f_{\text {tun }}$ is the tuning angular frequency.

Figure 13 depicts the loss factor of the system for the two circuits. The resonant circuit creates a frequency band where the system's response is largely attenuated, this band can be shifted by simply modifying the value of inductance. This behavior is attributed to the impedance mismatch in the mechanical properties of the assembly. The value of the tuning frequency affects also the 
shape of the attenuation bands. The loss factor associated to the resonant circuit tuned at $3000 \mathrm{~Hz}$ (green bars) exhibits a range of effectiveness wider than the loss factor associated to the resonant circuit tuned at $1000 \mathrm{~Hz}$ (red bars). On the other hand the circuit tuned at $1000 \mathrm{~Hz}$ shows a larger loss factor in a very narrow band, in Figure 13 this phenomenon is less evident because the frequency-band averaging performed on the response tends to mitigate this effect occurring in a very narrow band. The negative capacitance circuit (blue bars) provides a good mitigation effect over a wider frequency band keeping larger values over the whole frequency band of interest. For instance an increase of 0.005 is observed from $250 \mathrm{~Hz}$ to $2250 \mathrm{~Hz}$. The wave filtering effect usually observed in control devices based on negative capacitance circuits is less evident in resonant circuit. In Figure 13 the kinetic energy distribution in the lower region of the plate is compared the three considered cases. For the circuit tuned at $1000 \mathrm{~Hz}$ the filtering effect appears slightly larger than the negative capacitance circuit only in the vicinity of the tuning frequency. The same trend is observed for the resonant circuit tuned at $3000 \mathrm{~Hz}$. The best performances are provided by the negative capacitance circuit, in this case a larger amount of energy is confined in the bottom region of the plate.

\section{Conclusions}

A periodic configuration of negative capacitance shunted piezoelectric patches is used to control vibrations of a freely suspended aluminum plate.

Numerical investigations shown that the tuning capabilities of the negative capacitance networks can be effectively used to obtain a broadband control effect. The behavior of the smart plate is characterized by two different control mechanisms: the negative capacitance branch eliminates the parasitic effect due to the intrinsic capacitance of the piezoelectric ceramic enhancing the attenuation capabilities of the smart structure and the periodic lattice of piezoelectric actuator selectively filters the incident waves preventing the energy to pass through the smart interface.

The validated model allows to define some design criteria such as the choice of the electrical components, the size and the shape of the periodic lattice of piezoelectric patches. Some aspects of the robust design is also considered analyzing the effect of a randomly varying negative capacitance ratio on the overall performances of the system.

At the end of this paper the negative capacitance control strategy is compared to the classical solution based on resonant circuits with the aim to show that the first solution offers numerous advantages such as the design simplicity and the effectiveness over a wider frequencies range. In fact, the resonant circuit paradigm requires the tuning of each circuit around a specific frequency that usually coincide with a structure's resonance. This event implies the correct knowledge of the controlled structure, if the considered structure undergoes a change the overall performance of the control system can be sharply decreased. By using negative capacitance circuits this phenomenon is less evident since the correct selection of the interface topology and circuit's parameters allows to effectively control the modes contained in a specific frequency band. Mover, the practical implementation of the negative capacitance strategy requires an electric circuit with simpler topology than the Antoniou circuit used to synthesize the inductive circuit. 


\section{Acknowledgments}

This study is a collaborative effort supported by the French Research Agency under grant number NT09-617542 (CALIOP Project) and ANR-11-LABX-0001-01 (Labex Action).

\section{References}

Alessandroni, S., dell'Isola, F., and Porfiri, M. (2002). A revival of electric analogs for vibrating mechanical systems aimed to their efficient control by PZT actuators. International Journal of Solids and Structures, 39(20):5295-5324.

Andreaus, U., dell'Isola, F., and Porfiri, M. (2004). Piezoelectric passive distributed controllers for beam flexural vibrations. Journal of Vibration and Control, 10(5):625-659.

Beck, B., Cunefare, K., Ruzzene, M., and Collet, M. (2011). Experimental analysis of a cantilever beam with a shunted piezoelectric periodic array. Journal of Intelligent Material Systems and Structures, 22(11):1177-1187.

Behrens, S., Fleming, A., and Moheimani, S. (2003). A broadband controller for shunt piezoelectric damping of structural vibration. Smart Materials and Structures, 12(1):18.

Bobrovnitskii, Y. (2004). Conservation laws in vibration theory. Doklady Physics, 49(3):171-174.

Casadei, F., Ruzzene, M., Dozio, L., and Cunefare, K. (2010). Broadband vibration control through periodic arrays of resonant shunts: experimental investigation on plates. Smart Materials and Structures, 19(1):150.

Collet, M., Ouisse, M., and Ichchou, M. (2012a). Structural energy flow optimization through adaptive shunted piezoelectric metacomposites. Journal of Intelligent Material Systems and Structures, 23(15):1661-1677.

Collet, M., Ouisse, M., Ichchou, M., and Ohayon, R. (2012b). Semi-active optimization of 2D wave dispersion into shunted piezo-composite systems for controlling acoustic interaction. Smart Materials and Structures, 21(9):94.

dell'Isola, F., Maurini, C., and Porfiri, M. (2004). Passive damping of beam vibrations through distributed electric networks and piezoelectric transducers: prototype design and experimental validation. Smart Materials and Structures, 13(2):299.

Fleming, A., Behrens, S., and Moheimani, S. (2000). Synthetic impedance for implementation of piezoelectric shunt-damping circuits. Electronics Letters, 36(18):1525-1526.

Forward, R. (1979). Electronic damping of vibrations in optical structures. Applied Optics, 18(5):690-697.

Hagood, N. and von Flotow, A. (1991). Damping of structural vibrations with piezoelectric materials and passive electrical networks. Journal of Sound and Vibration, 146(2):243-268.

Hollkamp, J. (1994). Multimodal passive vibration suppression with piezoelectric materials and resonant shunts. Journal of Intelligent Material Systems and Structures, 5(1):49-57. 
Hughes, T. (2000). The finite element method linear static and dynamic finite element analysis. Dover Publications.

Livet, S., Collet, M., Berthillier, M., Pierrick, J., and Cote, J. (2011). Structural multi-modal damping by optimizing shunted piezoelectric transducers. European Journal of Computational Mechanics, 20(1-4):73-102.

Maurini, C., dell'Isola, F., and Del Vescovo, D. (2004). Comparison of piezoelectronic networks acting as distributed vibration absorbers. Mechanical Systems and Signal Processing, 18(5):12431271.

Mead, D. (1996). Wave propagation in continuous periodic structures: research contributions from southampton, 1964-1995. Journal of Sound and Vibration, 190(3):495-524.

Park, C. and Baz, A. (2005). Vibration control of beams with negative capacitive shunting of interdigital electrode piezoceramics. Journal of Vibration and Control, 11(3):331-346.

Park, J. and Palumbo, D. (2004). A new approach to identify optimal properties of shunting elements for maximum damping of structural vibration using piezoelectric patches. In Proc. Active 2004 conference.

Royer, D. and Dieulesaint, E. (2000). Elastic waves in solids. Springer.

Spadoni, A., Ruzzene, M., and Cunefare, K. (2009). Vibration and wave propagation control of plates with periodic arrays of shunted piezoelectric patches. Journal of Intelligent Material Systems and Structures, 20(8):979-990.

Thorp, O., Ruzzene, M., and Baz, A. (2005). Attenuation of wave propagation in fluid-loaded shells with periodic shunted piezoelectric rings. Smart Materials and Structures, 14(4):594.

Vidoli, S. and dell'Isola, F. (2001). Vibration control in plates by uniformly distributed PZT actuators interconnected via electric networks. European Journal of Mechanics - A/Solids, 20(3):435456.

Wu, S. (1999). Multiple PZT transducers implemented with multiple-mode piezoelectric shunting for passive vibration damping. In Proc. SPIE 3672, Smart Structures and Materials, pages $112-122$. 